\title{
Características epidemiológicas, clínicas, tratamiento y pronóstico de los pacientes con diagnóstico de síndrome coronario agudo en unidad especializada
}

\author{
Epidemiologieal and elinieal charaeteristies, \\ prognosis and treatment of patients with aeute- \\ eoronary syndrome in a chest pain unit
}

\author{
Juan Carlos Chavarriaga, Javier Beltrán, Juan Manuel Senior, \\ Andrés Fernández, Arturo Rodríguez, Juan Manuel Toro • \\ Medellín (Colombia)
}

\section{Resumen}

Introduccion: el síndrome coronario agudo es una de las principales causas de consulta en los servicios de urgencias y cardiología, con una alta tasa de mortalidad y con altos costos para la sociedad. Existe muy poca información con respecto a población Latinoaméricana y de Colombia en los estudios multicentricos internacionales. Se convierte en un reto conocer a profundidad las características epidemiológicas, clínicas, de laboratorio, escalas de riesgo usadas, hallazgos angiográficos, tratamiento instaurado y mortalidad en pacientes que han sido atendidos en un hospital de cuarto nivel de la ciudad de Medellín.

Material y métodos: estudio epidemiológico, observacional, descriptivo, longitudinal, retrospectivo tipo serie de casos. La población de estudio corresponde a los pacientes mayores de 18 años de edad, que consultaron a la Unidad de Dolor Torácico del Hospital Universitario San Vicente Fundación con diagnóstico de síndrome coronario agudo en un periodo de tres meses. Los pacientes continuaron su tratamiento según las recomendaciones y guías tanto internacionales como locales para dolor torácico. Luego de seis meses del síndrome coronario agudo, se realizó un contacto con el paciente por varios medios, evaluaciones de consulta externa, historia clínica y llamada telefónica. Se obtuvo información de su evolución clínica, estado funcional y complicaciones incluyendo reintervención, hospitalización o muerte, de igual forma se evaluó la adherencia al manejo médico.

Resultados: un total de 154 pacientes fueron analizados, $30 \%(n=47)$ por angina inestable, $37 \%$ $(\mathrm{n}=56)$ por IAMST y $33 \%(\mathrm{n}=51)$ por IAMNST. El promedio de edad fue de $62 \pm 13$ años, hombres 54\% ( $\mathrm{n}=83)$. Los factores de riesgo asociados fueron: Hipertensión arterial 66\% ( $\mathrm{n}=101)$, Dislipidemia $23 \%(\mathrm{n}=35)$, Diabetes Mellitus 18\% ( $\mathrm{n}=27)$, Obesidad 5,1\% $(\mathrm{n}=8)$ y tabaquismo $52 \%(\mathrm{n}=80)$. El total de cateterismos realizados fue de 129 ; normales el $21.8 \%(n=28)$ y lesiones obstructivas significativas en $78.2 \%(n=101)$. De los pacientes con IAMST $(n=56)$, sólo el $28 \%$ recibieron reperfusión primaria. La mortalidad intrahospitalaria fue del $7 \%(n=11)$. Luego de seis meses de seguimiento se presentaron seis muertes más (12\%). La adherencia a los tratamientos farmacológicos a los seis meses estuvo entre 54 y $86 \%$. De los pacientes que egresaron con orden de rehabilitación cardiaca, sólo el 3\% la habían realizado luego de 6 meses del egreso.

Conclusiones: los pacientes que ingresan a la unidad de dolor torácico del Hospital San Vicente Fundación con síndrome coronario agudo presentan unas características epidemiológicas de base similares a las reportadas en la literatura médica. Sin embargo, la mortalidad es superior. Se documentó un bajo número de pacientes con IAMST que pudieron recibir tratamiento de reperfusión. También se encontró baja adherencia al manejo farmacológico y a la rehabilitación cardiaca, esto posiblemente en relación a acceso limitado a los servicios de salud de alta complejidad de forma oportuna. (Acta Med Colomb 2014; 39: 00-00).

Palabras clave: síndrome coronario agudo, estratificación de riesgo, puntaje TIMI, puntaje GRACE
Dr. Juan Carlos Chavarriaga Zapata: Especialista en Medicina Interna, Especialista en Cardiologia Clínica, Universidad de Antioquia; Dr. Javier Ricardo Beltrán Bohórquez: Especialista en Medicina Interna y Cardiologia, Especialista en Cardiologia Intervencionista, Universidad de Antioquia; Dr. Juan Manuel Senior Sánchez: Especialista en Medicina Interna y Cardiologia, Especialista en Cardiologia Intervencionista, Coordinador Posgrado Cardiologia Clínica e Intervencionista, Universidad de Antioquia; Dr. Andres Fernández Cadavid: Especialista en Medicina Interna y Cardiologia, Especialista en Cardiologia Intervencionista, Hospital Universitario San Vicente de Paul Fundación; Dr. Arturo Rodríguez Dimuro: Especialista en Medicina Interna y Cardiologia, Especialista en Cardiologia Intervencionista, Hospital Universitario San Vicente de Paul Fundación; Dr. Juan Manuel Toro Escobar: Especialista en Medicina Interna, Especialista en Epidemiologia Clínica, Universidad de Antioquia. Medellín (Colombia).

Correspondencia. Juan Manuel Dr. Senior Sanchez, Coordinador Posgrado Cardiologia Clínica y Cardiologia Intervencionista, Facultad de Medicina Universidad de Antioquia. Medellín (Colombia).

E-mail: mmbt@une.net.co

Recibido: 7/VI/2013 Aceptado: 17/XII/2013 


\begin{abstract}

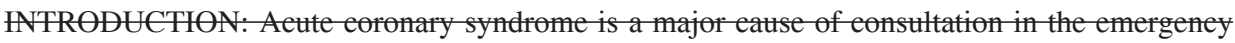
department andeardiology, with a high mortality rate and high eosts to publie health. There is very little information regarding Colombia population and Latin Ameriea in the international multieentrie trials and registries. It beeomes a challenge to know in depth the epidemiologieal, elinieal and laboratory eharaeteristies, risk stratifieation, angiographie findings, treatment, and on mortality in patients who have been treated at a hospital in fourth level of the eity of Medellin.

MATERHALS AND METHЮ円S: Epidemiologieal, observational, deseriptive, longittedinal, retrospective ease series. The study poptlation eorresponds to patients over 18 years of age, who eonsulted the ehest pain unit of the Hospital Universitario San Vieente Fundaeión with aeute eoronary syndrome in a 3 months period. The patients eontinted their treatment as reeommended by both international and toeal guidelines for ehest pain. After six months of aeute eoronary syndrome every patient was evaluated by various means: outpatient evaluations, medieal history and phone eall. Information was obtained from their elinieal and funetional statts and eomplieations ineluding reintervention, hospitalization or teath, simitarly assessed adherenee to medieal management.

RESULTS: A total of 154 patients were analyzed, $30 \%(n=47)$ for unstable angina, $37 \%(n=56)$ for STEMI and $33 \%(\mathrm{n}=51)$ for NSTEMI. The average age was $62 \pm 13$ years, men $54 \%(\mathrm{n}=83)$. The risk faetors were: hypertension $66 \%(\mathrm{n}=101)$, dyslipidemia $23 \%(\mathrm{n}=35)$, diabetes mellittus $18 \%$ $(\mathrm{n}=27)$, obesity $5.1 \%(\mathrm{n}=8)$ and smoking $52 \%(\mathrm{n}=80) .129$ eatheterizations was performed in the eatheterization laboratory; normal $21.8 \%(\mathrm{n}=28)$ and signifieant obstruetive lesions in $78.2 \%$ ( $\mathrm{n}=$ 101). $56(28 \%)$ patients with STEMI reeeived reperfusion therapies. In-hospital mortality was $7 \%$ (n $=11$ ). After six months of follow up were 6 deaths more $(12 \%)$. Adherenee to pharmacologieal treatments at six months was between 54-86\%. Only $3 \%$ of patients performed a regular program of eardiae rehabilitation, six months after diseharge.

CONCLUSIONS: Patients admitted to the ehest pain unit of the Hospital Universitario San Vieente Fundación presented with aeute coronary syndrome based epidemiologieal charaeteristies similar to those reported in the literature. However, the mortality rate is higher. We doeumented a low number of patients with STEMI who reeeived reperfusion therapy. We also found poor adherenee to pharmacologieal management and eardiae rehabilitation, this possibly related to limited aeeess to health serviees of high eomplexity in our public health system. (Acta Med Colomb 2014; 39: 00-00).
\end{abstract}

Key words: aeute eoronary syndrome, risk stratifieation, TIMI seore, GRACE seore.

\section{Introducción}

El avance en el diagnostico y tratamiento de los eventos coronarios agudos ha beneficiado a la población, por el incremento de la sobrevida y la mejoría en la calidad de vida después de un episodio índice, debido a la utilización de estrategias como la creación de unidades de dolor torácico, cuyo objetivo ha sido conocer la frecuencia de los factores de riesgo cardiovascular en la población local, agilizar la atención de los pacientes, incrementar la certeza diagnóstica, establecer la adherencia a las guías de manejo vigentes, disminuir costos y mejorar el pronóstico (1-3).

Conocemos las estadísticas y publicaciones de países desarrollados, sin embargo los datos de Latinoamérica son mucho más difíciles de obtener a pesar de presentar en los últimos años, una mayor incidencia y prevalencia de enfermedad coronaria. Entre otros estudios, contamos con la información suministrada por el registro CARMELA (4), que es un análisis sobre la prevalencia de los factores de riesgo cardiovascular realizado en siete ciudades latinoamericanas (1553 colombianos de 11550 pacientes latinos), en los cuales se documentó que los factores de riesgo más comunes fueron tabaquismo (30\%), obesidad (23\%), síndrome metabólico (20\%), hipertensión arterial (18\%), hipercolesterolemia (14\%), diabetes mellitus (7\%) y placa carotídea en el $8 \%$. También se cuenta con el estudio INTERHEART (5), el cual evaluó la presencia de factores de riesgo en pacientes hospitalizados por infarto agudo del miocardio y los comparó con una población "sana". Se desarrolló en 52 países e incluyó a 1888 pacientes latinoamericanos y 1200 controles, (cerca de 3000 personas, $11 \%$ del total mundial) de los cuales 800 fueron colombianos y sus resultados demostraron alta prevalencia de factores de riesgo cardiovascular en todo el mundo, incremento del riesgo de un evento coronario entre 2 a 4 veces si fuma o si es diabético, hipertenso o dislipidémico; si se combinan factores el riesgo aumenta, hasta 13 veces si fuma y es diabético o hipertenso; si además tiene niveles lipídicos inadecuados, el riesgo aumenta 42 veces. $\mathrm{El}$ análisis univariado del riesgo demuestra que los niveles inadecuados de lípidos son responsables de casi 50\% de los infartos agudo de miocardio (IAM) en el mundo; el tabaco da cuenta de un tercio y los factores psicosociales, como estrés y depresión, de casi un tercio más; la obesidad representa el 
$20 \%$. El consumo de alcohol, el ejercicio y el consumo diario de frutas y vegetales son factores protectores y la ausencia de ellos constituye un factor de riesgo

Por último el registro internacional más importante hasta el momento es el GRACE (6-8) que cuenta con más de 102000 pacientes, de los cuales un porcentaje no despreciable son latinoamericanos. En este se ha reportado la incidencia de factores de riesgo cardiovascular así: Diabetes mellitus $23 \%$, hipertensión arterial $58 \%$, infarto de miocardio previo $32 \%$, dislipidemia $45 \%$ y tabaquismo $58 \%$. La mortalidad establecida en el registro GRACE (6) es del 7\% para el IAMST, 4\% para el IAMNST y del 3\% para la AI.

Según la organización Mundial de la salud (OMS) cada dos segundos se produce una muerte por enfermedad cardiovascular en el mundo (9), cada cinco segundos un infarto de miocardio y cada seis segundos un evento cerebrovascular, esto sitúa la cardiopatía isquémica como responsable del treinta por ciento de muertes en todo el mundo constituyendo la principal causa de fallecimiento (10). Estados Unidos reporta casi 700000 personas fallecidas anualmente por enfermedades cardiovasculares (11). En España en el año 2000 se reportaron 125723 muertes, lo que supone el 35\% de todas las defunciones, con una tasa bruta de mortalidad de 315 por 100.000 habitantes (12).

En Colombia existe información basada en estudios que tienen en cuenta los certificados de defunción y muestran que entre el año 1970 y 2000 no ha disminuido significativamente la mortalidad, en 1970 era de 94 muertes por 100000 habitantes y para el 2000 de 93 por 100000 habitantes (13). Esta información de la población general del país, revela la gravedad de la enfermedad coronaria y la sitúa como la principal causa de muerte en el país, mayor aún que los homicidios que ocupan el segundo lugar. Sin embargo, no se cuenta con información acerca de la mortalidad del síndrome coronario agudo. De acuerdo con las estadísticas publicadas por el ministerio de la protección social en el informe sobre la situación de salud en Colombia, indicadores de salud 2007, la enfermedad isquémica del corazón es la primera causa de muerte tanto en hombres como en mujeres mayores de 45 años de edad o más, e incluso supera las muertes violentas o el cáncer, combinados (14). Por ende conocer el comportamiento de esta enfermedad en nuestra población se convierte en pilar fundamental para establecer políticas de salud que permitan mejorar el resultado en el tratamiento en la población afectada y disminuir las secuelas a largo plazo (15).

\section{Material y metodos}

Estudio epidemiológico, observacional, descriptivo, longitudinal, retrospectivo tipo serie de casos. La población de estudio corresponde a los pacientes mayores de 18 años de edad, que consultan a la Unidad de Dolor Torácico del Hospital Universitario San Vicente Fundación con diagnóstico de síndrome coronario agudo en un periodo de tres meses, en forma consecutiva.
Se estableció el diagnóstico de síndrome coronario agudo de acuerdo a los cambios eléctricos y la presencia de marcadores de necrosis. Se clasificaron como síndrome coronario agudo con elevación del segmento ST o infarto agudo de miocardio con elevación del segmento ST (IAMST) y síndrome coronario agudo sin elevación del segmento ST (SCANST), dentro del cual se incluyeron al infarto agudo de miocardio sin elevación del ST (IAMNST) y a la angina inestable (AI) (16-18).

Se utilizaron escalas para predecir el pronóstico de los pacientes con IAMNST o AI, como la escala de riesgo TIMI $(19,20)$ y GRACE (6). La escala TIMI tiene 7 variables, se suma un punto por cada una que se encuentre presente, dando un máximo de 7 (Tabla 1) (http://www.timi.org/). Basados en este resultado los pacientes se clasifican en tres grupos: puntaje de 0 a 2, riesgo bajo (desenlace entre 4.7-8.3\%) de morir o presentar complicaciones como infarto de miocardio o necesidad de revascularización a 14 días; riesgo intermedio (desenlace adverso entre 13.2 a $19.9 \%$ ) con puntajes de 3 y 4 y riesgo alto (desenlace adverso entre 26.2 a $40.9 \%$ ) con puntajes entre 5 y 7 .

La escala de riesgo GRACE predice el riesgo de muerte o infarto agudo de miocardio intrahospitalario y a seis meses, se realiza al ingreso teniendo en cuenta las siguientes variables: edad, frecuencia cardiaca, presencia de falla cardiaca, paro cardíaco al ingreso, presión arterial sistólica, alteración del segmento ST, elevación de las enzimas cardiacas y creatinina.

(http://www.outcomes-umassmed.org/grace/acs_risk/ acs_risk_content.html).

También se utiliza la escala GRACE al egreso para predecir mortalidad y reinfarto a 6 meses.

Estas escalas incluyen la determinación de marcadores séricos de necrosis miocárdica como la troponina I (21-24). Otros marcadores séricos fueron incluidos como la proteína C reactiva (PCR) (25-28) y el Péptido Natriurético cerebral (BNP) (29-32).

Los pacientes con SCANST con elevación de las troponinas y/o riesgo alto o intermedio en la escala de TIMI o GRACE deberían ser llevados a una estrategia invasiva temprana, en otras palabras realizar estudio angiográfico en las siguientes 24-72 horas (33), al igual que todos los pacientes con IAMST para reperfusión primaria (primeras doce horas) o para estratificación si llegaron fuera de ven-

Tabla 1. Escala de Riesgo TIMI

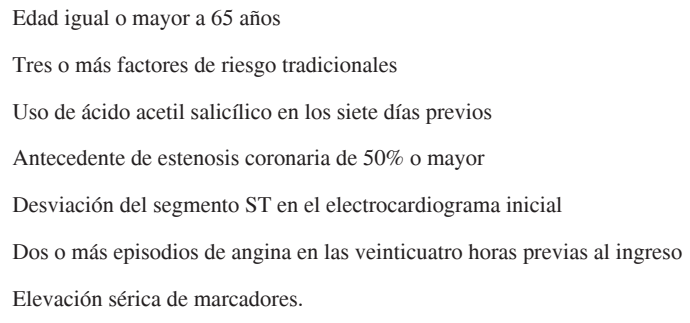


tana. Aquellos pacientes con riesgo bajo serían candidatos a una estratificación no invasiva.

Todos los pacientes deben iniciar un programa de rehabilitación cardiaca que tiene como eje principal la educación, cambios de hábitos así como metas claras, tangibles en cuanto a colesterol, presión arterial, peso y actividad física (34). Las enfermedades asociadas deben ser controladas lo antes posible, en especial la hipertensión arterial, la diabetes mellitus, la hipercolesterolemia y alteraciones tiroideas entre otras. El tabaquismo debe ser intervenido desde un principio y se puede convertir en el objetivo principal del manejo postinfarto (35).

Se revisaron los registros de la historia clínica de cada paciente donde se constataron los datos demográficos, síntomas de presentación, hallazgos electrocardiográficos, antecedentes médicos, laboratorios, evaluación, manejo y complicaciones del tratamiento médico e intervencionista. Luego de seis meses del síndrome coronario agudo, se realizó un contacto con el paciente por varios medios, evaluaciones por consulta externa, revisión de historia clínica o en su defecto por llamada telefónica. Se obtuvo información de su evolución clínica, estado funcional y complicaciones incluyendo reintervención, hospitalización o muerte, de igual forma se evaluó la adherencia al manejo médico.

Se excluyeron todos los pacientes con otros diagnósticos, casos que no correspondían al período en estudio y que además no contaran con un expediente clínico en el archivo del servicio de estadística del Hospital.

\section{Análisis estadístico}

Se digitó y depuró en Excel versión 2003, se procesó la información en el paquete estadístico para ciencias sociales para Windows SPSS versión 15 y Epi Dat 3.1. Se describieron las variables cualitativas mediante distribuciones de frecuencias y porcentuales, variables cuantitativas con medidas de tendencia central como el promedio y mediana y medidas de dispersión como la desviación estándar y rango intercuartílico.

\section{Resultados}

Se registraron 154 pacientes que ingresaron a la unidad de dolor torácico del Hospital Universitario San Vicente de Paúl con dolor torácico e impresión diagnostica de síndrome coronario agudo. De estos, 83 (53.9\%) fueron hombres y $71(46.1 \%)$ mujeres. El $71 \%$ de los pacientes procedían del área metropolitana de la ciudad de Medellín y el $28 \%$ fueron remitidos del área rural de diferentes municipios de Antioquia. La edad promedio fue de 62 años (entre 23 y 93 años).

Los factores de riesgo encontrados en esta población fueron: hipertensión arterial en 102 (66\%), diabetes mellitus en 27 (17\%), dislipidemia en 36 (23\%), tabaquismo en $80(52 \%)$ y obesidad en $8(5 \%)$ de los pacientes. Cinco (3\%) pacientes habían sido sometidos a cirugía de revascularización quirúrgica con puentes, 28 (18\%) pacientes presentaba antecedente personal de enfermedad coronaria e intervencionismo previo con stent y $11(7 \%)$ tenían historia familiar de enfermedad coronaria; $48(32 \%)$ pacientes habían presentado angina previamente, falla cardíaca 7 (5\%), infarto previo $18(12 \%)$, enfermedad cerebrovascular o isquemia cerebral transitoria $5(3 \%)$, EPOC $10(6 \%)$ y falla renal crónica 14 (9\%) (Tabla 2).

Los episodios fueron clasificados como síndrome coronario agudo sin elevación del segmento ST el 63.6\%: AI en el $30.5 \%$ de los pacientes e infarto agudo de miocardio sin elevación del segmento ST en 33.1\%; IAMST en el 36.4\% de los casos. Los síntomas fueron de características típicas en el 55\% de los pacientes. El electrocardiograma mostró cambios en la pared anterior en $31 \%$ de los casos, inferior en el $21.4 \%$, lateral en el $6 \%$, posterior en el $1.9 \%$ y en el $38.3 \%$ se presentaron en 2 o más (Tabla 3 ).

Tabla 2. Características basales de los pacientes con SCA que ingresaron a la Unidad de Dolor Torácico.

\begin{tabular}{|lc|}
\hline Característica & $\mathrm{n}=154$ \\
\hline Edad, años & \\
$\quad$ Media (DE) & $62(13)$ \\
\hline Sexo & \\
Hombres & $83(53.9 \%)$ \\
Mujeres & $71(46.1 \%)$ \\
\hline Historia médica previa & \\
Hipertensión arterial & $101(65.6 \%)$ \\
Diabetes mellitus & $27(17.5 \%)$ \\
Dislipidemia & $35(22.7 \%)$ \\
Fumadores activos & $80(51.9 \%)$ \\
Obesidad & $8(5.1 \%)$ \\
Antecedente familiar de EC & $11(7.1 \%)$ \\
Angina previa & $48(31.1 \%)$ \\
Infarto de miocardio previo & $19(12.3 \%)$ \\
Cirugía de puentes coronarios & $5(3.2 \%)$ \\
Intervención percutánea previa & $27(17.5 \%$ \\
Falla cardiaca & $7(4.5 \%)$ \\
Insuficiencia renal & $14(9.1 \%)$ \\
Enfermedad cerebrovascular & $5(3.2 \%)$ \\
Enfermedad pulmonar obstructiva crónica & $10(6.5 \%)$ \\
\hline
\end{tabular}

Tabla 3. Características clínicas y de laboratorio de los pacientes con SCA que ingresaron a la Unidad de Dolor Torácico.

\begin{tabular}{|lc|}
\hline Clasificación del evento coronario & \\
Angina inestable & $47(30.5 \%)$ \\
Infarto agudo de miocardio con elevación ST & $56(36.4 \%)$ \\
Infarto agudo de miocardio sin elevación ST & $51(33,1 \%)$ \\
\hline Laboratorios (DE) & \\
Troponina (ng/ml) & $4.3(2.1)$ \\
Glicemia (mg/dl) & $122(64)$ \\
Colesterol total (mg/dl) & $193(51)$ \\
Colesterol HDL (mg/dl) & $41(13)$ \\
Colesterol LDL (mg/dl) & $121(38)$ \\
Triglicéridos (mg/dl) & $174(93)$ \\
Péptido natriurético cerebral (pg/ml) & $592(84)$ \\
Proteína C reactiva (mg/L) & $2,7(0,38)$ \\
Creatinina (mg/dL) & $1,07(0.31)$ \\
\hline
\end{tabular}


Las escalas de evaluación de riesgo TIMI y GRACE en los pacientes con angina inestable y con infarto agudo de miocardio sin elevación del ST ( $\mathrm{n}=98)$ mostraron el siguiente perfil de riesgo: TIMI riesgo bajo 30 pacientes (31\%), TIMI riesgo intermedio 38 pacientes (38.8\%), TIMI riesgo alto $30(30.6 \%)$ (Tabla 4).

La estratificación de riesgo según escala GRACE en el grupo de pacientes con IAMNST y AI se observa en la Tabla 5.

De 68 pacientes con SCANST clasificados como riesgo TIMI bajo e intermedio, la estratificación inicial se realizó por pruebas no invasivas en $30(44 \%)$, de la siguiente manera: con ecocardiografía de estrés a 19 (28\%), con prueba de esfuerzo convencional a $7(10 \%)$, con perfusión miocárdica por medicina nuclear a $4(6 \%)$; la estrategia invasiva se utilizó en $13(19 \%)$ pacientes y a $25(37 \%)$ no se les realizó ninguna prueba.

De los 154 pacientes a $129(83 \%)$ se llevaron a coronariografía, mientras que a $25(17 \%)$ no se les realizó ninguna estratificación adicional luego de su ingreso. En los pacientes con IAMST $(\mathrm{n}=56)$ se realizó reperfusión primaria a 18 (32.1\%) pacientes, de los cuales a $16(28.4 \%)$ pacientes fue con angioplastia primaria e implantación de stent y a 2 (3.7\%) con trombolisis; uno con activador tisular del plasminógeno y el otro con estreptoquinasa. De los 16 pacientes con IAMST que recibieron tratamiento con angioplastia primaria e implantación de stent, se administró tirofibán a $9(56 \%)$ y se les practicó trombectomía con catéter a 7 (44\%). El tiempo desde el inicio de los síntomas hasta el procedimiento de

Tabla 4. Características del evento índice.

\begin{tabular}{|cc|}
\hline Características del evento coronario actual & \\
\hline Angina típica & $85(55.2 \%)$ \\
\hline Tiempo de evolución del dolor hasta consultar & 105 \\
\hline Fracción de expulsión (DE) & $52.2 \%(16.4 \%)$ \\
\hline Puntaje TIMI SCA no ST & \\
Riesgo bajo (1-2) & $30(30.6 \%)$ \\
Riesgo intermedio (3-4) & $38(38,8 \%)$ \\
Riesgo alto (5-7) & $30(30.6 \%)$ \\
\hline Clasificación Killip & $138(89.7 \%)$ \\
Killip I & $10(6.5 \%)$ \\
Killip II & $3(1.9 \%)$ \\
Killip III & $3(1.9 \%)$ \\
Killip IV & \\
\hline
\end{tabular}

Tabla 5. Estratificación de riesgo con escala GRACE en pacientes con IAMNST y AI.

\begin{tabular}{|c|c|c|}
\hline GRACE & Riesgo & Frecuencia \\
\hline$\leq 108$ & Riesgo bajo & $63(64.3 \%)$ \\
$109-140$ & Riesgo Intermedio & $26(26.5 \%)$ \\
140 & Riesgo alto & $9(9,2 \%)$ \\
\hline Total & & $\mathbf{9 8}$ \\
\hline
\end{tabular}

angioplastia primaria e implantación de stent fue en promedio 5.9 horas. En el resto de pacientes con IAMST no se realizó terapia de reperfusión primaria por ingresar a la institución fuera de ventana terapéutica (más de 12 horas).

En la fase hospitalaria se utilizó clopidogrel en el 93\% de los pacientes, betabloqueadores en el $75.9 \%$, ASA en $92 \%$, IECA en el $68 \%$, morfina al $11 \%$ y nitroglicerina a $14 \%$. A $17(11 \%)$ pacientes se les administró Tirofibán (Inhibidor de Glicoproteína IIb/IIIa), enoxaparina a 131 (85\%), fondaparinux a $3(2 \%)$ y heparina no fraccionada al $9(6 \%)$.

Las complicaciones del SCA encontradas fueron: 20 pacientes con falla cardíaca de los cuales 11 requirieron manejo en UCI por choque cardiogénico, 2 presentaron fibrilación ventricular y 1 taquicardia ventricular.

Se realizó angiografía coronaria a 129 pacientes, documentando coronarias epicárdicas sin lesiones obstructivas significativas en 28 pacientes (21.8\%), en 101 (78.2\%) se encontraron diferentes grados de lesión así: enfermedad de un vaso en 33 pacientes ( $32.8 \%$ de los enfermos), dos vasos en $24(23.7 \%)$, tres en 39 pacientes $(38.6 \%)$ y compromiso del tronco principal izquierdo en 5 pacientes (4.9\%). Como complicaciones del cateterismo se documentaron en 3 pacientes hematomas inguinales y en 10 sangrado menor, además hubo un evento isquémico cerebral y otro hemorrágico y 7 casos de falla renal aguda.

De los 101 pacientes en los que se encontró enfermedad coronaria, se les realizó angioplastia con stent a 74 (73.3\%), de los cuales a $55(74.3 \%)$ se les implantó stent convencional, a $19(25.7 \%)$ stent liberador de medicamento, $13(12.8 \%)$ fueron intervenidos con cirugía de puentes coronarios y $14(13.9 \%)$ pacientes no revascularizables por ningún método.

De los 154 pacientes estudiados murieron en la fase hospitalaria 11 (7\%). De los 143 pacientes dados de alta salieron formulados con: ASA $119(83 \%)$, IECA 108 (75\%), betabloqueador $108(75 \%)$, estatinas $112(78 \%)$ y clopidogrel a $87(60 \%)$ pacientes.

\section{Evaluación 6 meses después del alta}

A los 6 meses del alta se evaluaron los registros de 124 pacientes, de los cuales 11 fallecieron durante la hospitalización inicial, por lo tanto el análisis del seguimiento se hizo sobre 113 pacientes. Esto constituye una pérdida de seguimiento del $19.4 \%$. Luego de seis meses de seguimiento, 89 pacientes recibían tratamiento con estatinas, 89 con betabloqueadores y 83 con IECA. La clase funcional ambulatoria fue I de la NYHA en $56 \%$, II en $33 \%$, III en $4 \%$ y IV en el $1 \%$. Sólo el $3 \%$ de los pacientes ingresaron a un programa de rehabilitación cardiaca. El $7 \%$ tuvo reingresos y 6 pacientes (5\%) murieron en esos 6 meses de seguimiento, lo que da una mortalidad global a 6 meses de 17 pacientes (12\%). De los 74 pacientes a los que se les implantó un stent, 4 pacientes $(6 \%)$, presentaron síntomas anginosos y se documentó reestenosis. No se documentaron trombosis del stent. 


\section{Discusión}

Este estudio constituye una valiosa documentación de las características de un grupo de pacientes evaluados en una unidad especializada de una institución de alta complejidad y de referencia, teniendo en cuenta que el $28 \%$ de los pacientes fueron remitidos de diferentes municipios del área rural de Antioquia. La información obtenida puede ser una de las primeras en nuestro medio, aunque investigadores del registro GRACE recolectaron datos de algunos centros en nuestro país y en la ciudad, estos no son ampliamente conocidos y solo fueron publicados en formato de resumen.

Algunas características encontradas en nuestra población son similares a las reportadas en la literatura; al compararlas con las del registro GRACE se evidencia población etaria similar $65 \pm 13$ GRACE vs $62 \pm 13$ serie actual, sin embargo es importante resaltar que la distribución depende de la prevalencia de los factores de riesgo coronario y de variables genéticas, encontrando diferencias importantes de acuerdo a la población estudiada $(36,37)$.

Es importante enfatizar que se encontraron diferencias relevantes en la presencia de algunos factores de riesgo coronario, siendo los más frecuentes hipertensión arterial (65.6\%) y el tabaquismo (51.9\%), con menor participación de la dislipidemia (22.7\%), a diferencia del registro GRACE y del registro Europeo, en los cuales el tabaquismo tiene un peso menor y la dislipidemia se convierte en marcador importante. Sin embargo un aspecto relevante es la relación del evento coronario agudo con los factores de riesgo tradicionales, corroborando los resultados del subanálisis para Latinoamérica del estudio INTERHEART, lo que podría reafirmar las políticas de salud en el control de estos (38).

Llama la atención que, a pesar de ser una institución de alta complejidad, el porcentaje de pacientes con antecedente de revascularización miocárdica, quirúrgica o percutánea, es relativamente bajo (21\%) en comparación al GRACE (28\%), similar al registro europeo (21.5\%) pero mayor que en la población árabe de acuerdo al registro Gulf RACE (39). En contraposición encontramos un número alto de pacientes con antecedentes de falla cardiaca, insuficiencia renal crónica, enfermedad cerebrovascular y enfermedad pulmonar obstructiva crónica, que puede ser explicado por el tipo de pacientes atendidos en un centro de referencia para enfermedades renales y cardiovasculares.

El patrón de presentación de los SCA ha cambiado en los últimos años, observándose preponderancia de los SCANST sobre el IMEST, posiblemente por el avance en el tratamiento de la enfermedad coronaria y el efecto de la terapia de revascularización percutánea o quirúrgica. En nuestra población encontramos que los SCANST representan el 63.6\% de los casos, AI en el $30.5 \%$ de los pacientes e infarto agudo de miocardio sin elevación del segmento ST en 33.1\%; similar a lo reportado por los investigadores del National Registry of Myocardial Infarction en Estados Unidos y en otros estudios en los cuales se distribuyen de forma homogénea en tercios cada una de las tres clasificaciones (40).
El perfil de riesgo de la población de estudio varía significativamente de acuerdo al puntaje o escala utilizado, encontrando un alto porcentaje de pacientes de alto riesgo (30.6\%) de acuerdo al TIMI y mucho menor $(9,2 \%)$ con el GRACE. Podría ser explicado por sesgos introducidos en la recolección de los datos, dado el aspecto retrospectivo del estudio, sin embargo se utilizaron registros bastantes confiables de la base de datos de la Unidad de Dolor Torácico, o relacionados con la falta de validación de las dos escalas en nuestra población (41). Comparado con lo reportado por otros estudios, en países latinoamericanos como Argentina, la distribución difiere por la inclusión de un porcentaje importante de pacientes de bajo riesgo (30.6\%) en nuestra población (42), posiblemente por la clasificación inadecuada de los episodios, teniendo en cuenta el perfil de las características de la población, el tratamiento intervencionista y la mortalidad.

De los resultados que más cuestionamientos genera es la baja proporción de pacientes con IAMST que recibieron reperfusión primaria, dada la remisión fuera de ventana. Solo se reperfundieron el $32.1 \%$ de los pacientes en las primeras doce horas, al resto se les realizó angiografía coronaria como estratificación invasiva posinfarto, muy por debajo de lo reportado en las diferentes series, incluyendo países con economías poco solidas (43). Hecho que explica parcialmente que el $44 \%$ de los pacientes a los seis meses estuviera en clase funcional NYHA $\geq$ II. Esta claramente demostrado que la reperfusión primaria con angioplastia e implantación de stent supera en resultados a la terapia fibrinolítica (44), sin embargo también es evidente que su efecto depende del tiempo en que se restaure el flujo sanguíneo en el vaso culpable, por lo que esta ultima estrategia se convierte en un pilar fundamental del tratamiento en pacientes con IAMST que no puedan ser remitidos en forma oportuna (45). Es recomendable adelantar estudios adicionales que permitan establecer las causas de estos retrasos y generar políticas de salud tendientes a reducir el tiempo de presentación de los infartos y poder ofrecer terapias de reperfusión oportunas que lleven a los pacientes a mejorar la calidad de vida y a disminuir la mortalidad observada en este estudio.

La mortalidad hospitalaria fue alta. Al tomar todos los pacientes se encontró que a los 6 meses la mortalidad fue del $12 \%$, comparado con $4.7 \%$ encontrado en el registro GRACE durante el mismo lapso, incluso podría ser mayor dado que la tasa de pérdidas del seguimiento alcanzó el $19 \%$. Se podrían esbozar algunas explicaciones como la tasa de pacientes que no fueron reperfundidos en la fase inicial, el desarrollo de falla cardiaca y choque cardiogénico con requerimiento de manejo en cuidados intensivos y la pobre adherencia a las diferentes terapias farmacológicas y no farmacológicas (54\% con clopidogrel) a 6 meses. En los pacientes con IAMST, también se encontró una mortalidad muy alta (14.2\%) a los 6 meses comparado con lo reportado en la literatura (5\%). En este caso es muy probable que la falta de revascularización en el $68 \%$ de los pacientes haya contribuido a incrementar la mortalidad. 
Llama la atención la baja adherencia ambulatoria a los medicamentos y en especial al uso de clopiodogrel, 54\% de los pacientes intervenidos durante un SCA recibían antiagregación dual a los 6 meses, y el escaso número de pacientes que tuvo acceso a un programa de rehabilitación cardiaca, lo que altera el reintegro del paciente a su vida familiar, social y laboral.

También preocupa que no haya sido posible contactar a 30 pacientes a los seis meses. Esto representa una pérdida de seguimiento de $19 \%$. A pesar de una búsqueda exhaustiva en los archivos y en los teléfonos suministrados no fue posible contactarlos. Esto puede indicar un mal registro de los datos de los pacientes o que por tratarse de una población muy pobre y vulnerable, se encuentre un número importante de pacientes en situación de indigencia, pacientes desplazados, que luego no pueden contactarse. No se realizó análisis de sensibilidad de estos casos y solo se describe la mortalidad de la población que se pudo hacer seguimiento. Es posible que un número significativo de pacientes que no pudieron seguirse, hayan fallecido, esto agravaría aún más la situación y el pronóstico de esta población con síndrome coronario agudo.

\section{Limitaciones del estudio}

Esta población tiene un importante sesgo de selección al tratarse de pacientes evaluados en un hospital de alta complejidad, centro de remisión de diferentes partes de la ciudad y de la región, algunas de ellas muy distantes. Además recibe, en su gran mayoría, a personas de bajos recursos, con nivel de escolaridad mínima. Sin embargo, dicha selección nos permite comparar los resultados de esta población con la publicada en países industrializados.

La recolección de 154 pacientes en un lapso de tres meses, se constituye en una muestra pequeña en relación con la magnitud del problema, lo cual impide validar de forma categórica los puntajes de riesgo pronóstico TIMI y GRACE y establecer prevalencias o incidencias.

El tipo de diseño del estudio, descriptivo y retrospectivo, lleva a dificultades en la recolección de los datos y seguimiento de los pacientes. A pesar de esta limitante y después de una exhaustiva búsqueda, fue posible recuperar información en 113 pacientes a los 6 meses. La situación social y cultural contribuye a ésta disminución del número de personas.

\section{Conclusiones}

Los pacientes que ingresan a la unidad de dolor torácico del Hospital Universitario San Vicente de Paul con síndrome coronario agudo presentan unas características epidemiológicas, factores de riesgo cardiovascular y antecedentes de base particulares, en algunos casos similares a las reportadas en la literatura médica.

Existen evidentes diferencias en la estratificación de riesgo por las dos escalas utilizadas, lo que hace imperativo realizar un estudio al respecto.
El tratamiento médico de los pacientes con infarto agudo del miocardio sin elevación del segmento ST y con angina inestable está de acuerdo con lo recomendado por las guías nacionales e internacionales. En el caso del infarto con elevación del segmento encontramos muy poco pacientes se les ofrece terapia de reperfusión primaria por tiempos de llegada al Hospital muy superiores a 12 horas, lo que se asoció a una mortalidad muy elevada.

Se encontró baja adherencia al manejo farmacológico y a la rehabilitación cardiaca después del alta hospitalaria.

\section{Declaración de fuentes de financiación y posibles conflictos de interés}

El presente estudio fue financiado con recursos propios de la Seccion de Cardiologia de la Facultad de Medicina de la Universidad de Antioquia.

No hay conflictos de interés.

\section{Referencias}

1. McGovern PG, Jacobs DR Jr, Shahar E, Arnett DK, Folsom AR, Blackburn $\mathbf{H}$, et al. Trends in acute coronary heart disease mortality, morbidity, and medical care from 1985 through 1997: the Minnesota heart survey. Circulation. 2001 Jul 3; 104(1): 19-24.

2. Rosamond WD, Chambless LE, Folsom AR, Cooper LS, Conwill DE, Clegg $\mathbf{L}$, et al. Trends in the incidence of myocardial infarction and in mortality due to coronary heart disease, 1987 to 1994. N Engl J Med 1998 Sep 24; 339(13): 861-867.

3. Eslick G. Usefulness of Chest Pain Character and Location as Diagnostic Indicators of an Acute Coronary Syndrome. Am J Card. 2005 May; 95(10): 1228-1231.

4. Schargrodsky H, Hernández-Hernández R, Champagne BM, Silva H, Vinueza R, Silva Ayçaguer LC, et al. CARMELA: assessment of cardiovascular risk in seven Latin American cities. Am J Med 2008 Ene; 121(1): 58-65.

5. Yusuf S, Hawken S, Ounpuu S, Dans T, Avezum A, Lanas F, et al. Effect of potentially modifiable risk factors associated with myocardial infarction in 52 countries (the INTERHEART study): case-control study. Lancet 2004 Sep 11; 364(9438): 937-952.

6. Eagle KA, Lim MJ, Dabbous OH, Pieper KS, Goldberg RJ, Van de Werf F, et al. A validated prediction model for all forms of acute coronary syndrome: estimating the risk of 6-month postdischarge death in an international registry. JAMA. 2004 Jun 9; 291(22): 2727-2733.

7. Goldberg RJ, Steg PG, Sadiq I, Granger CB, Jackson EA, Budaj A, et al. Extent of, and factors associated with, delay to hospital presentation in patients with acute coronary disease (the GRACE registry). Am J Cardiol 2002 Abr 1; 89(7): 791-796.

8. GRACE Investigators. Rationale and design of the GRACE (Global Registry of Acute Coronary Events) Project: a multinational registry of patients hospitalized with acute coronary syndromes. Am Heart J 2001 Feb; 141(2): 190-199.

9. Velázquez-Monroy O, Rosas Peralta M, Lara Esqueda A, Pastelín Hernández G, Sànchez-Castillo C, Attie F, et al. [Prevalence and interrelations of noncommunicable chronic diseases and cardiovascular risk factors in Mexico. Final outcomes from the National Health Survey 2000]. Arch Cardiol Mex 2003 Mar; 73(1): 62-77.

10. Murray CJ, Lopez AD. Mortality by cause for eight regions of the world: Global Burden of Disease Study. Lancet. 1997 May 3;349(9061):1269-1276.

11. Department of health and human services. The burden of chronic diseases and their risk factors - National and state perspectives. 2004;1:1-182.

12. R. Boix, S. Cañellas, J. Almazán, E. Mortalidad cardiovascular en España. Año 2000. Boletin epidemiológico semanal 2003; 11(21): 241-252.

13. Rodríguez T, Malvezzi M, Chatenoud L, Bosetti C, Levi F, Negri E, et al. Trends in mortality from coronary heart and cerebrovascular diseases in the Americas: 1970-2000. Heart 2006 Abr; 92(4): 453-460.

14. Beltrán JR, Beltrán R, Caicedo VM. Guías colombianas de cardiología: Síndrome coronario agudo sin elevación del ST. Rev Col Card. 2008; 15: 141-232.

15. Kushner FG, Hand M, Smith SC, King SB, Anderson JL, Antman EM, et al. 2009 Focused Updates: ACC/AHA Guidelines for the Management of Patients With ST-Elevation Myocardial Infarction (Updating the 2004 Guideline and 2007 Focused Update) and ACC/AHA/SCAI Guidelines on Percutaneous Coronary Intervention (Updating the 2005 Guideline and 2007 Focused Update). J Ame Coll Card 2009 Dic; 54(23): 2205-2241. 
16. Thygesen K, Alpert JS, White HD. Universal definition of myocardial infarction. J Am Coll Cardiol 2007 Nov 27; 50(22): 2173-2195.

17. Beltrán JR, Beltrán R, Bohórquez R. Guías colombianas de cardiología sindrome coronario agudo con elevación del ST. Revista Colombiana de Cardiología 2010; 17: 121-275.

18. Antman EM, Hand M, Armstrong PW, Bates ER, Green LA, Halasyamani LK, et al. 2007 Focused Update of the ACC/AHA 2004 Guidelines for the Management of Patients With ST-Elevation Myocardial Infarction: a report of the American College of Cardiology/American Heart Association Task Force on Practice Guidelines: developed in collaboration With the Canadian Cardiovascular Society endorsed by the American Academy of Family Physicians: 2007 Writing Group to Review New Evidence and Update the ACC/AHA 2004 Guidelines for the Management of Patients With ST-Elevation Myocardial Infarction, Writing on Behalf of the 2004 Writing Committee. Circulation 2008 Ene 15; 117(2): 296-329.

19. Schweitzer P, Keller S. The role of the initial 12-lead ECG in risk stratification of patients with acute coronary syndrome. Bratisl Lek Listy 2001; 102(9): 406-411.

20. Antman EM, Cohen M, Bernink PJ, McCabe CH, Horacek T, Papuchis G, et al. The TIMI risk score for unstable angina/non-ST elevation MI: A method for prognostication and therapeutic decision making. JAMA 2000 Ago 16; 284(7): 835-842.

21. Antman EM, Tanasijevic MJ, Thompson B, Schactman M, McCabe CH, Cannon CP, et al. Cardiac-specific troponin I levels to predict the risk of mortality in patients with acute coronary syndromes. $N$ Engl J Med 1996 Oct 31; 335(18): 1342-1349.

22. Ohman EM, Armstrong PW, Christenson RH, Granger CB, Katus HA, Hamm CW, et al. Cardiac troponin $\mathrm{T}$ levels for risk stratification in acute myocardial ischemia. GUSTO IIA Investigators. $N$ Engl J Med 1996 Oct 31; 335(18): 1333 1341 .

23. Kontos MC, Anderson FP, Alimard R, Ornato JP, Tatum JL, Jesse RL. Ability of troponin I to predict cardiac events in patients admitted from the emergency department. J Am Coll Cardiol 2000 Nov 15; 36(6): 1818-1823.

24. Lindahl B, Toss H, Siegbahn A, Venge P, Wallentin L. Markers of myocardial damage and inflammation in relation to long-term mortality in unstable coronary artery disease. FRISC Study Group. Fragmin during Instability in Coronary Artery Disease. N Engl J Med 2000 Oct 19; 343(16): 1139-1147.

25. Danesh J, Wheeler JG, Hirschfield GM, Eda S, Eiriksdottir G, Rumley A, et al. C-reactive protein and other circulating markers of inflammation in the prediction of coronary heart disease. N Engl J Med 2004 Abr 1; 350(14): 1387-1397.

26. Ridker PM. C-reactive protein and the prediction of cardiovascular events among those at intermediate risk: moving an inflammatory hypothesis toward consensus. $J$ Am Coll Cardiol 2007 May 29; 49(21): 2129-2138.

27. Morrow DA, Rifai N, Antman EM, Weiner DL, McCabe CH, Cannon CP, et al. C-reactive protein is a potent predictor of mortality independently of and in combination with troponin T in acute coronary syndromes: a TIMI 11 A substudy. Thrombolysis in Myocardial Infarction. J Am Coll Cardiol 1998 Jun; 31(7): 14601465 .

28. Bassuk SS, Rifai N, Ridker PM. High-sensitivity C-reactive protein: clinical importance. Curr Probl Cardiol 2004 Ago; 29(8): 439-493.

29. Richards M, Nicholls MG, Espiner EA, Lainchbury JG, Troughton RW, Elliott J, et al. Comparison of B-type natriuretic peptides for assessment of cardiac function and prognosis in stable ischemic heart disease. J Am Coll Cardiol 2006 Ene $3 ;$ 47(1): 52-60.
30. Vasan RS. Biomarkers of cardiovascular disease: molecular basis and practical considerations. Circulation 2006 May 16; 113(19): 2335-2362.

31. Felker GM, Petersen JW, Mark DB. Natriuretic peptides in the diagnosis and management of heart failure. CMAJ 2006 Sep 12; 175(6): 611-617.

32. Jernberg T, James S, Lindahl B, Stridsberg M, Venge P, Wallentin L. NTproBNP in unstable coronary artery disease--experiences from the FAST, GUSTO IV and FRISC II trials. Eur J Heart Fail 2004 Mar 15; 6(3): 319-325.

33. de Winter RJ, Windhausen F, Cornel JH, Dunselman PHJM, Janus CL, Bendermacher PEF, et al. Early invasive versus selectively invasive management for acute coronary syndromes. N Engl J Med 2005 Sep 15; 353(11): 1095-1104.

34. Witt BJ, Jacobsen SJ, Weston SA, Killian JM, Meverden RA, Allison TG, et al. Cardiac rehabilitation after myocardial infarction in the community. J. Am. Coll. Cardiol. 2004 Sep 1; 44(5): 988-996.

35. Nissen SE, Tuzcu EM, Schoenhagen P, Crowe T, Sasiela WJ, Tsai J, et al. Statin therapy, LDL cholesterol, C-reactive protein, and coronary artery disease. N Engl J Med 2005 Ene 6; 352(1): 29-38.

36 Awad H,Zubaid M,Alsheikh-Ali A, Suwaidi J, Anderson F, Gore J, Goldeber R. Comparison of characteristics, management practices, and outcomes of patients between the global registry and the Gulf registry of acute coronary syndromes. Am J Cardiol 2011; 108 (9):1252-1258.

37 Mandelzweig L, Battler A, Bueno H, Danchin N, Filipatos G, Gitt A et al. The second Euro Heart Survey on acute coronary syndromes: characteristics, treatment, and outcomes of patients with ACS in Europe and the meditarrean Basin in 2004. Eur Heart J 2006; 27(19): 2285-2293.

38 Lanas F, Avezum A, Bautista L, Diaz R, Luna M, Islam S et al. Risk Factors for Acute Myocardial Infarction in Latin America The INTERHEART Latin American Study. Circulation 2007; 115: 1067-1074.

39 Zubaid M, Rashed WA, Almahmeed W, Al-Lawati J, Sulaiman K, Al-Motarreb A et al. Management and outcomes of Middle Eastern patients admitted wit acute coronary syndromes in the Gulf Registry of acute coronary events. Acto Cardiol 2009; 64: 439-446.

40 Rogers W, Frederick P, Stoehr E, Canto J, ornaro J, Gibson C et al. Trends in presenting characteristics and hospital mortality among patients with ST elevation and non ST elevation myocardial oinfarction in the national registry of myocardial infarction from 1990 to 2006. Am Heart J 2008; 156(6): 1026-1034.

41 Filipiak K, Koltowski L, Grabowski M, Karpinski G, Glowczynska R, Huczek $\mathbf{Z}$ et al. Prospective Comparison of the 5 Most Popular Risk Scores in Clinical Use for Unselected Patients With Acute Coronary Syndrome. Circ J 2011; 75 167-173.

42 Bagur R, Urinovsky F, Contreras A, Estrada C. Validación del score de riesgo TIMI para pacientes con síndrome coronario agudo sin elevación del segmento ST. MEDICINA (Buenos Aires) 2009; 69 (5): 526-528

43 Andrikopoulus G, Tzeis S, Mantas I, Olympios C, Kitsiou A, Kartalis A et al. Epidemiological characteristics and in hospital management of acute coronary syndrome patients in Greece: results from the TARGET Study. Hellenic J Cardiol 2012; 53: 33-40

44 Keeley E, Boura J, Grines C. Primary angioplasty versus intravenous thrombolytic therapy for acute myocardial infarction: a quantitative review of 23 randomised trials. Lancet 2003; 361: 13-20.

45 Tarantini G, Razzolini R, Napodano M, Bilato C, Ramondo A, Iliceto S. Acceptable reperfusion delay to prefer primary angioplasty over fibrin-specific thrombolytic therapy is affected (mainly) by the patient's mortality risk: $1 \mathrm{~h}$ does not fit all. Eur Heart J 2010; 31: 676-83. 